\title{
The Growth of Entire Functions Defined by Laplace-Stieltjes Transforms Related to Proximate Order and Approximation
}

\author{
Wen Ju Tang, ${ }^{1}$ Jian Chen, ${ }^{2}$ and Hong Yan $X u i^{3}$ \\ ${ }^{1}$ Department of Informatics and Engineering, Jingdezhen Ceramic Institute, Jingdezhen, Jiangxi 333403, China \\ ${ }^{2}$ Xinyu No. 4 Middle School of Jiangxi Province, Xinyu, Jiangxi 338004, China \\ ${ }^{3}$ School of Mathematics and Computer Science, Shangrao Normal University, Shangrao, Jiangxi 334001, China \\ Correspondence should be addressed to Hong Yan Xu; xhyhhh@126.com
}

Received 14 February 2020; Accepted 11 March 2020; Published 9 April 2020

Academic Editor: Kehe Zhu

Copyright (c) 2020 Wen Ju Tang et al. This is an open access article distributed under the Creative Commons Attribution License, which permits unrestricted use, distribution, and reproduction in any medium, provided the original work is properly cited.

\begin{abstract}
In this article, we discuss the growth of entire functions represented by Laplace-Stieltjes transform converges on the whole complex plane and obtain some equivalence conditions about proximate growth of Laplace-Stieltjes transforms with finite order and infinite order. In addition, we also investigate the approximation of Laplace-Stieltjes transform with the proximate order and obtain some results containing the proximate growth order, the error, $A_{n}^{*}$, and $\lambda_{n}$, which are the extension and improvement of the previous theorems given by Luo and Kong and Singhal and Srivastava.
\end{abstract}

\section{Introduction}

Our main aim of this paper is to investigate some problems about the growth and approximation of entire functions represented by Laplace-Stieltjes transforms which converge on the whole complex plane. Consider Laplace-Stieltjes transforms

$$
F(s)=\int_{0}^{+\infty} e^{s x} \mathrm{~d} \alpha(x), \quad s=\sigma+i t, \sigma, t \in R,
$$

where $\alpha(x)$ is a bounded variation on any finite interval $[0, Y](0<Y<+\infty)$. For Laplace-Stieltjes transform (1), set

$$
A_{n}^{*}=\sup _{\lambda_{n}<x \leq \lambda_{n+1},-\infty<t<+\infty}\left|\int_{\lambda_{n}}^{x} e^{i t y} \mathrm{~d} \alpha(y)\right|,
$$

where the sequence $\left\{\lambda_{n}\right\}_{0}^{\infty}$ satisfies

$$
0 \leq \lambda_{1}<\lambda_{2}<\cdots<\lambda_{n}<\cdots, \lambda_{n} \longrightarrow \infty \text { as } n \longrightarrow \infty
$$

$$
\begin{gathered}
\limsup _{n \longrightarrow+\infty} \frac{\log A_{n}^{*}}{\lambda_{n}}=-\infty, \\
\limsup _{n \longrightarrow+\infty} \frac{\log n}{\lambda_{n}}=D<+\infty, \\
\limsup _{n \longrightarrow+\infty}\left(\lambda_{n+1}-\lambda_{n}\right)=h<+\infty,
\end{gathered}
$$

similar to the method in [1], and by using the Valiron-Knopp-Bohr formula, then it yields $\sigma_{u}^{F}=+\infty$, that is, $F(s)$ is an entire function in the whole plane. We denote $\bar{L}_{\beta}$ to be a class of all the functions $F(s)$ of form (1) which are analytic in the half plane $\mathfrak{R} s<\beta(-\infty<\beta<\infty)$, and the sequence $\left\{\lambda_{n}\right\}$ satisfies (3) and (5), and denote $L_{\infty}$ to be the class of all the functions $F(s)$ of form (1) which are analytic in the whole plane $\Re s<+\infty$, and the sequence $\left\{\lambda_{n}\right\}$ satisfies (3), (5), and (4). Thus, if $-\infty<\beta<+\infty$ and $F(s) \in L_{\infty}$, then $F(s) \in \bar{L}_{\beta}$.

By Widder [2], if $\alpha(t)$ is absolutely continuous, then $F(s)$ is the classical Laplace integral form:

$$
F(s)=\int_{0}^{\infty} e^{s t} g(t) \mathrm{d} t
$$


if $\alpha(t)$ is a step-function and a sequence $\left\{\lambda_{n}\right\}_{0}^{\infty}$ satisfies (3), and

$$
\alpha(x)= \begin{cases}a_{1}+a_{2}+\cdots+a_{n}, & \lambda_{n} \leq x<\lambda_{n+1} ; \\ 0, & x=0,\end{cases}
$$

then $F(s)$ becomes a Dirichlet series:

$$
F(s)=\sum_{n=1}^{\infty} a_{n} e^{\lambda_{n} s}, \quad s=\sigma+i t, \sigma, t \in R,
$$

where $a_{n}$ are nonzero complex numbers; if $\alpha(t)$ is an increasing continuous function which is not absolutely continuous, then integral (1) defines a class of functions $F(s)$ which cannot be expressed either in form (6) or (8) (see [2]).

In 1963, Yu [1] first proved the Valiron-Knopp-Bohr formula of the associated abscissas of bounded convergence, absolute convergence, and uniform convergence of Laplace-Stieltjes transform when $s=-z$.

Theorem 1. If the sequence $\left\{\lambda_{n}\right\}$ satisfies (3) and (5), then Laplace-Stieltjes transform (1) satisfies

$$
\limsup _{n \longrightarrow+\infty} \frac{\log B_{n}^{*}}{\lambda_{n}} \leq \sigma_{u}^{F} \leq \limsup _{n \longrightarrow+\infty} \frac{\log B_{n}^{*}}{\lambda_{n}}+\limsup _{n \longrightarrow+\infty} \frac{\log n}{\lambda_{n}},
$$

where $\sigma_{u}^{F}$ is called the abscissa of uniformly convergence of $F(s)$, and

$$
B_{n}^{*}=\sup _{\lambda_{n}<x \leq \lambda_{n+1},-\infty<t<+\infty}\left|\int_{\lambda_{n}}^{x} e^{-i t y} \mathrm{~d} \alpha(y)\right| .
$$

In addition, $\mathrm{Yu}$ [1] introduced the concept of the order of $G(z)=F(-s)$ to estimate the growth of the maximal molecule $M_{u}(\sigma, G)$ and the maximal term $\mu(\sigma, G)$ and gave the relation between the Borel line and the order of entire functions represented by Laplace-Stieltjes transform converge in the whole complex plane. After his wonderful works, considerable attention has been paid to the growth, and the value distribution of the functions represented by Laplace-Stieltjes transform or Dirichlet series converges in the half plane or the whole complex plane in the field of complex analysis (see [3-24]).

Set

$$
\begin{aligned}
\mu(\sigma, F) & =\max _{n \in N}\left\{A_{n}^{*} e^{\lambda_{n} \sigma}\right\}(\sigma<+\infty), \\
M_{u}(\sigma, F) & =\sup _{0<x<+\infty,-\infty<t<+\infty}\left|\int_{0}^{x} e^{(\sigma+i t) y} \mathrm{~d} \alpha(y)\right| .
\end{aligned}
$$

In view of $M_{u}(\sigma, F) \longrightarrow+\infty$ as $\sigma \longrightarrow+\infty$, in order to estimate the growth of $F(s)$ precisely, we usual use the concepts of order and type as follows.

Definition 1. If $F(s) \in L_{\infty}$ and

$$
\limsup _{\sigma \longrightarrow+\infty} \frac{\log ^{+} \log ^{+} M_{u}(\sigma, F)}{\sigma}=\rho, \quad 0 \leq \rho \leq+\infty,
$$

then it is said that $F(s)$ is of order $\rho$ in the whole plane, where $\log ^{+} x=\max \{\log x, 0\}$. Furthermore, if $\rho \in(0,+\infty)$, the type of $F(s)$ is defined by

$$
\limsup _{\sigma \longrightarrow+\infty} \frac{\log ^{+} M_{u}(\sigma, F)}{e^{\sigma \rho}}=T, \quad 0 \leq T \leq+\infty .
$$

Let $F(s)$ be of order $\rho$ in the whole plane and $0<\rho<+\infty$, and let $\rho(\sigma)\left(\sigma>\sigma_{0}\right)$ be a nonnegative, continuous, and monotonous function, and it has a left-hand derivative and right-hand derivative in every $\sigma\left(>\sigma_{0}\right)$ such that

$$
\begin{array}{r}
\lim _{\sigma \longrightarrow+\infty} \rho(\sigma)=\rho, \\
\lim _{\sigma \longrightarrow+\infty} \sigma \rho^{\prime}(\sigma)=0,
\end{array}
$$

then $\rho(\sigma)$ is called the proximate order; furthermore, if

$$
\limsup _{\sigma \longrightarrow+\infty} \frac{\log ^{+} M_{u}(\sigma, F)}{e^{\sigma \rho(\sigma)}}=T,
$$

then $T$ will be called the proximate type of $F(s)$ with respect to proximate order $\rho(\sigma)$.

Set $r=e^{\sigma}$, and let $t=\psi(r)=r^{\rho(\log r)}=e^{\sigma \rho(\sigma)}$ and $r=\varphi(t)$ be two reciprocally inverse functions. Then, we obtain the following theorem.

Theorem 2. If Laplace-Stieltjes transform $F(s) \in L_{\infty}$ and is of proximate order $\rho(\sigma)$ and order $\rho(0<\rho<+\infty)$, then

$$
\tau \leq T \leq e^{D \rho} \tau,
$$

where

$$
\tau=\limsup _{n \longrightarrow+\infty} \frac{1}{\rho e}\left[\varphi\left(\lambda_{n}\right)\left(A_{n}^{*}\right)^{1 / \lambda_{n}}\right]^{\rho} .
$$

Furthermore, if the sequence $\left\{\lambda_{n}\right\}$ satisfies

$$
\liminf _{n \longrightarrow+\infty}\left(\lambda_{n+1}-\lambda_{n}\right)=h^{\prime}>0,
$$

then

$$
T=\tau=\limsup _{n \longrightarrow+\infty} \frac{1}{\rho e}\left[\varphi\left(\lambda_{n}\right)\left(A_{n}^{*}\right)^{1 / \lambda_{n}}\right]^{\rho}
$$

In order to estimate the growth of Laplace-Stieltjes transform of order $\rho=+\infty$ precisely, we will use some concepts of the $p$-order as follows.

Definition 2. If $F(s) \in L_{\infty}, p \geq 2$ is a positive integer, and

$$
\underset{\sigma \longrightarrow+\infty}{\limsup } \frac{\log _{p+1} M_{u}(\sigma, F)}{\sigma}=\rho_{p}, \quad 0 \leq \rho(p) \leq+\infty,
$$

then we call $F(s)$ is of $p$-order $\rho_{p}$ in the whole plane, where $\log _{p+1} x=\log _{p} \log x$ for $p \in \mathbb{N}_{+}$.

Let $F(s)$ be of $p$-order $\rho_{p}$ in the whole plane and $0<\rho_{p}<+\infty$, similar to the proximate type of $F(s)$ with respect to proximate order $\rho(\sigma)$; we can give the proximate type of $F(s)$ with respect to proximate order of $p$-order $\rho_{p}$ as follows. Let $\rho_{p}(\sigma)\left(\sigma>\sigma_{0}\right)$ be a nonnegative, continuous, and monotonous function, and it has a lefthand derivative and right-hand derivative in every $\sigma\left(>\sigma_{0}\right)$ such that 


$$
\begin{aligned}
& \lim _{\sigma \longrightarrow+\infty} \rho_{p}(\sigma)=\rho_{p}, \\
& \lim _{\sigma \longrightarrow+\infty} \sigma \rho_{p}^{\prime}(\sigma)=0,
\end{aligned}
$$

then $\rho_{p}(\sigma)$ is called the $p$-proximate order; furthermore, if

$$
\underset{\sigma \longrightarrow+\infty}{\limsup } \frac{\log _{p} M_{u}(\sigma, F)}{e^{\sigma \rho_{p}(\sigma)}}=T_{p}
$$

then $T_{p}$ will be called the proximate type of $F(s)$ with respect to $p$-proximate order $\rho_{p}(\sigma)$.

Set $r=e^{\sigma}$, and let $t=\psi_{p}(r)=r^{\rho_{p}(\log r)}=e^{\sigma \rho_{p}(\sigma)}$ and $r=$ $\varphi_{p}(t)$ be two reciprocally inverse functions. Then, we obtain the following theorem.

Theorem 3. If Laplace-Stieltjes transform $F(s) \in L_{\infty}$ and is of p-proximate order $\rho_{p}(\sigma)$ and $\rho_{p}\left(0<\rho_{p}<+\infty\right), p \geq 2$, then

$$
\tau_{p} \leq T_{p} \leq e^{D \rho_{p}} \tau_{p}
$$

where

$$
\tau_{p}=\limsup _{n \longrightarrow+\infty}\left[\varphi_{p}\left(\log _{p-1} \lambda_{n}\right)\left(A_{n}^{*}\right)^{1 / \lambda_{n}}\right]^{\rho_{p}} .
$$

Furthermore, if the sequence $\lambda_{n}$ satisfies (18), then

$$
T_{p}=\tau_{p}=\limsup _{n \longrightarrow+\infty}\left[\varphi_{p}\left(\log _{p-1} \lambda_{n}\right)\left(A_{n}^{*}\right)^{1 / \lambda_{n}}\right]^{\rho_{p}} .
$$

The other purpose of this article is to study the approximation of the entire function represented by Laplace-Stieltjes transform converges in the whole plane. When Laplace-Stieltjes transform (1) satisfies $A_{k}^{*} \neq 0$ and $A_{n}^{*}=0$ for $n \geq k+1$, then $F(s)$ will be called an exponential polynomial of degree $k$ usually denoted by $p_{k}$, i.e., $p_{k}(s)=\int_{0}^{\lambda_{k}} \exp (s y) \mathrm{d} \alpha(y)$. When we choose a suitable function $\alpha(y)$, the function $p_{k}(s)$ may be reduced to a polynomial in terms of $\exp \left(s \lambda_{i}\right)$, that is, $\sum_{i=1}^{k} b_{i} \exp \left(s \lambda_{i}\right)$. We also use $\Pi_{k}$ to denote the class of all exponential polynomial of degree almost $k$, that is,

$$
\Pi_{k}=\left\{\sum_{i=1}^{k} b_{i} \exp \left(s \lambda_{i}\right):\left(b_{1}, b_{2}, \ldots, b_{k}\right) \in \mathbb{C}^{k}\right\} .
$$

For $F(s) \in L_{\infty},-\infty<\beta<+\infty$, we denote by $E_{n}(F, \beta)$ the error in approximating the function $F(s)$ by exponential polynomials of degree $n$ in uniform norm as

$$
E_{n}(F, \beta)=\inf _{p \in \Pi_{n}}\|F-p\|_{\beta}, \quad n=1,2, \ldots,
$$

where

$$
\|F-p\|_{\beta}=\max _{-\infty<t<+\infty}|F(\beta+i t)-p(\beta+i t)| .
$$

Recently, Singhal and Srivastava [15] and Xu and Liu [25] studied the approximation on Laplace-Stieltjes transforms of finite order and obtained the following theorems.

Theorem 4 (see [15]). If Laplace-Stieltjes transform $F(s) \in L_{\infty}$ and is of order $\rho(0<\rho<\infty)$ and of type $T$, then for any real number $-\infty<\beta<+\infty$, we have

$$
\begin{aligned}
\rho & =\limsup _{n \longrightarrow+\infty} \frac{\lambda_{n} \log \lambda_{n}}{-\log E_{n-1}(F, \beta) \exp \left(-\beta \lambda_{n}\right)} \\
& =\limsup _{n \longrightarrow+\infty} \frac{\lambda_{n} \log \lambda_{n}}{-\log E_{n-1}(F, \beta)}, \\
T & =\limsup _{n \longrightarrow+\infty} \frac{\lambda_{n}}{\rho e}\left(E_{n-1}(F, \beta) \exp \left(-\beta \lambda_{n}\right)\right)^{\rho / \lambda_{n}} \\
& =\limsup _{n \longrightarrow+\infty} \frac{\lambda_{n}}{\rho \exp (\rho \beta+1)}\left(E_{n-1}(F, \beta)\right)^{\rho / \lambda_{n}} .
\end{aligned}
$$

Theorem 5 (see [25], Theorem 2.5). If the Laplace-Stieltjes transform $F(s) \in L_{\infty}$ and is of the lower order $\lambda(0 \leq \lambda \neq \rho<\infty)$ and if $\lambda_{n} \sim \lambda_{n+1}$, then for any real number $-\infty<\beta<+\infty$, we have

$$
\tau_{\lambda} \geq \liminf _{n \longrightarrow \infty}\left(\frac{\lambda_{n}}{e \lambda}\right)\left(E_{n-1}(F, \beta) \exp \left(-\beta \lambda_{n}\right)\right)^{\lambda / \lambda_{n}}, \quad\left(0 \leq \tau_{\lambda} \leq \infty\right) .
$$

Furthermore, there exists a positive integer $n_{0}$ such that

$$
\xi(n)=\frac{\log A_{n}^{*}-\log A_{n+1}^{*}}{\lambda_{n+1}-\lambda_{n}}
$$

forms a nondecreasing function of $n$ for $n>n_{0}$, and then we have

$$
\tau_{\lambda}=\liminf _{n \longrightarrow \infty}\left(\frac{\lambda_{n}}{e \lambda}\right)\left(E_{n-1}(F, \beta) \exp \left(-\beta \lambda_{n}\right)\right)^{\lambda / \lambda_{n}} \quad\left(0 \leq \tau_{\lambda} \leq \infty\right),
$$

i.e.,

$$
\exp (\beta \lambda) e \lambda \tau_{\lambda}=\liminf _{n \longrightarrow \infty} \lambda_{n}\left(E_{n-1}(F, \beta)\right)^{\lambda / \lambda_{n}}
$$

Corresponding to Theorems 2 and 3, we obtain the following results concerning the approximation.

Theorem 6. If Laplace-Stieltjes transform $F(s) \in L_{\infty}$ and is of proximate order $\rho(\sigma)$ and order $\rho(0<\rho<+\infty)$, then for any real number $-\infty<\beta<+\infty$, we have

$$
\tau_{a} \leq T \leq e^{D \rho} \tau_{a}
$$

where

$$
\tau_{a}=\limsup _{n \longrightarrow+\infty} \frac{1}{\rho \exp (\beta \rho+1)}\left[\varphi\left(\lambda_{n}\right)\left(E_{n-1}(F, \beta)\right)^{1 / \lambda_{n}}\right]^{\rho} .
$$

Furthermore, if the sequence $\lambda_{n}$ satisfies (18), then

$$
T=\tau_{a}=\limsup _{n \longrightarrow+\infty} \frac{1}{\rho \exp (\beta \rho+1)}\left[\varphi\left(\lambda_{n}\right)\left(E_{n-1}(F, \beta)\right)^{1 / \lambda_{n}}\right]^{\rho} .
$$

Theorem 7. If Laplace-Stieltjes transform $F(s) \in L_{\infty}$ and is of $p$-proximate order $\rho_{p}(\sigma)$ and $\rho_{p}\left(0<\rho_{p}<+\infty\right), p \geq 2$, then for any real number $-\infty<\beta<+\infty$, we have 


$$
\tau_{p a} \leq T_{p} \leq e^{D \rho_{p}} \tau_{p a}
$$

where

$$
\tau_{p a}=\limsup _{n \longrightarrow+\infty} \frac{1}{\exp \left(\beta \rho_{p}\right)}\left[\varphi_{p}\left(\log _{p-1} \lambda_{n}\right)\left(E_{n-1}(F, \beta)\right)^{1 / \lambda_{n}}\right]^{\rho_{p}} .
$$

Furthermore, if the sequence $\lambda_{n}$ satisfies (18), then $T_{p}=\tau_{p a}=\limsup _{n \longrightarrow+\infty} \frac{1}{\exp \left(\beta \rho_{p}\right)}\left[\varphi_{p}\left(\log _{p-1} \lambda_{n}\right)\left(E_{n-1}(F, \beta)\right)^{1 / \lambda_{n}}\right]^{\rho_{p}}$.

\section{Some Lemmas}

Lemma 1. For the functions $\psi, \varphi$, and $r=e^{\sigma}$, we have

$$
\begin{aligned}
& \lim _{\sigma \longrightarrow+\infty} \frac{r \psi^{\prime}(r)}{\psi(r)}=\rho, \\
& \lim _{t \rightarrow+\infty} \frac{t \varphi^{\prime}(t)}{\varphi(t)}=\frac{1}{\rho}, \\
& \lim _{\sigma \longrightarrow+\infty} \frac{\psi(k r)}{\psi(r)}=k^{\rho}, \\
& \lim _{t \rightarrow+\infty} \frac{\varphi(k t)}{\varphi(t)}=k^{1 / \rho}, \quad k>0 .
\end{aligned}
$$

Proof. Since $\left(t \varphi^{\prime}(t) / \varphi(t)\right)=\left(\psi(r) / r \psi^{\prime}(r)\right)$ and

$$
\lim _{\sigma \longrightarrow+\infty}\left(\frac{r \psi^{\prime}(r)}{\psi(r)}\right)=\lim _{\sigma \longrightarrow+\infty} \frac{e^{\sigma} e^{\sigma \rho(\sigma)}\left[\rho(\sigma)+\sigma \rho^{\prime}(\sigma)\right] e^{-\sigma}}{e^{\sigma \rho(\sigma)}}=\rho,
$$

then (40) follows. Moreover,

$$
\lim _{\sigma \longrightarrow+\infty} \frac{\psi(k r)}{\psi(r)}=\lim _{\sigma \longrightarrow+\infty} \frac{k^{\rho(\sigma)} \psi(r)}{\psi(r)}=k^{\rho} .
$$

Next, we prove the last equality. Since $\varphi(t)=e^{\sigma}$ and $t=e^{\sigma \rho(\sigma)}$, thus, it follows $\sigma \longrightarrow+\infty$ as $t \longrightarrow+\infty$ and $k t=k e^{\sigma \rho(\sigma)}$, and $\varphi(k t)=k^{(1 / \rho(\sigma))} e^{\sigma}$. Since $\lim _{\sigma \longrightarrow+\infty} \rho(\sigma)=$ $\rho$, then it yields

$$
\lim _{t \longrightarrow+\infty} \frac{\varphi(k t)}{\varphi(t)}=\lim _{\sigma \longrightarrow+\infty} \frac{k^{1 / \rho(\sigma)} e^{\sigma}}{e^{\sigma}}=\kappa^{1 / \rho}
$$

Therefore, this completes the proof of Lemma 1.

Remark 1. Obviously, the functions $\psi_{p}$ and $\varphi_{p}$ have the same conclusions as $\psi$ and $\varphi$ in Lemma 1 .

Lemma 2. Let $a, b>0$; then, the function

$$
h(r)=a \psi(r)-b \log r
$$

can obtain the minimum

$$
b\left\{\frac{1}{\rho(1+o(1))}-\log \varphi\left(\frac{b}{a \rho(1+o(1))}\right)\right\} \quad \text { in } \quad r=\varphi\left(\frac{b}{a \rho(1+o(1))}\right) .
$$

Proof. Let

$$
h^{\prime}(r)=a \psi^{\prime}(r)-\frac{b}{r}=\frac{1}{r}\left(\operatorname{ar} \psi^{\prime}(r)-b\right)=0 .
$$

Then, it means $a r \psi^{\prime}(r)-b=0$. In view of the first equality in (40), we deduce $b=a \rho(1+o(1)) \psi(r)$, that is,

$$
r=\varphi\left(\frac{b}{a \rho(1+o(1))}\right), \quad \text { as } \quad b \longrightarrow+\infty \text {. }
$$

We can see that, as the value of $r$ increases to the given value above, the value of $h(r)$ changes from a negative value to a positive value. Thus, $h(r)$ can obtain minimum

$$
b\left\{\frac{1}{\rho(1+o(1))}-\log \varphi\left(\frac{b}{a \rho(1+o(1))}\right)\right\}, \quad \text { in } \quad r=\varphi\left(\frac{b}{a \rho(1+o(1))}\right) \text {. }
$$

Therefore, this completes the proof of Lemma 2.

Lemma 3. Let $a>0$; then, the function

$$
g(t)=a t-t \log \varphi(t)
$$

can obtain the maximum

$$
\frac{1}{\rho}(1+o(1)) \psi\left(\frac{e^{a}}{e^{(1+o(1)) / \rho}}\right), \quad \text { in } \quad t=\psi\left(\frac{e^{a}}{e^{(1+o(1)) / \rho}}\right)(a \longrightarrow+\infty) \text {. }
$$

Proof. Let

$$
g^{\prime}(t)=a-\log \varphi(t)-\frac{t \varphi^{\prime}(t)}{\varphi(t)}=0 .
$$

Then, it follows from (40) that $a-\log \varphi(t)-1 / \rho(1+$ $o(1))=0$. In view of the first equality in (40), we deduce $\varphi(t)=\left(e^{a} / e^{(1+o(1) / \rho)}\right)$, that is,

$$
t=\psi\left(\frac{e^{a}}{e^{(1+o(1)) / \rho}}\right), \quad \text { as } \quad a \longrightarrow+\infty .
$$

It is easy to see that $g(t)$ obtain the maximum

$$
\psi\left(\frac{e^{a}}{e^{(1+o(1)) / \rho}}\right)\left[a-a+\frac{1}{\rho}(1+o(1))\right]=\frac{1}{\rho}(1+o(1)) \psi\left(\frac{e^{a}}{e^{(1+o(1)) / \rho}}\right),
$$$$
(a \longrightarrow+\infty), \quad \text { in } \quad t=\psi\left(\frac{e^{a}}{e^{(1+o(1)) / \rho}}\right)
$$

Therefore, this completes the proof of Lemma 3.

Lemma 4. If Laplace-Stieltjes transform $F(s) \in L_{\infty}$, for any $\sigma(0<\sigma<+\infty)$ and $\varepsilon(>0)$, we have

$$
\frac{1}{2} \mu(\sigma, F) \leq M_{u}(\sigma, F) \leq C e^{K \sigma} \mu(\sigma+D+\varepsilon, F),
$$


where $C$ and $K$ are constants.

Proof. We will adapt the method as in $\mathrm{Yu}[1]$ and Kong and Hong [7]. Set

$$
I(x ; \sigma+i t)=\int_{0}^{x} \exp \{(\sigma+i t) y\} \mathrm{d} \alpha(y)
$$

For $x>\lambda_{n}$, it follows

$$
\begin{aligned}
\int_{\lambda_{n}}^{x} \exp \{i t y\} \mathrm{d} \alpha(y)= & \int_{\lambda_{n}}^{x} \exp \{-\sigma y\} d_{y} I(y ; \sigma+i t) \\
= & \left.I(y ; \sigma+i t) \exp \{-\sigma y\}\right|_{\lambda_{n}} ^{x} \\
& +\sigma \int_{\lambda_{n}}^{x} \exp \{-\sigma y\} I(y ; \sigma+i t) \mathrm{d} y .
\end{aligned}
$$

Then, for any $\sigma(>0)$ and any $x \in\left(\lambda_{n}, \lambda_{n+1}\right]$, it yields

$$
\begin{aligned}
& \left|\int_{\lambda_{n}}^{x} \exp \{i t y\} \mathrm{d} \alpha(y)\right| \leq 2 M_{u}(\sigma, F)\left|\exp \left\{-\sigma \lambda_{n}\right\}-\exp \{-x \sigma\}\right| \\
& \quad \leq 2 M_{u}(\sigma, F) \exp \left\{-\lambda_{n} \sigma\right\}
\end{aligned}
$$

which implies

$$
\frac{1}{2} \mu(\sigma, F) \leq M_{u}(\sigma, F)
$$

On the contrary, for any $x>0$, it follows that there exists a positive integer $n \in \mathbb{N}_{+}$such that $\lambda_{n}<x \leq \lambda_{n+1}$. Thus, it follows

$$
\begin{aligned}
\int_{0}^{x} \exp \{(\sigma+i t) y\} \mathrm{d} \alpha(y)= & \sum_{k=1}^{n-1} \int_{\lambda_{k}}^{\lambda_{k+1}} \exp \{(\sigma+i t) y\} \mathrm{d} \alpha(y) \\
& +\int_{\lambda_{n}}^{x} \exp \{(\sigma+i t) y\} \mathrm{d} \alpha(y) .
\end{aligned}
$$

Set $I_{k}(x ;$ it $)=\int_{\lambda_{n}}^{x} \exp \{$ it $y\} \mathrm{d} \alpha(y),\left(\lambda_{k}<x \leq \lambda_{k+1}\right)$; then, for any real number $t$ and $\sigma>0$, it follows $\left|I_{k}(x ; i t)\right| \leq A_{k}^{*} \leq \mu(\sigma, F) e^{-\lambda_{k} \sigma}$. Thus, for any $\varepsilon \in(0,1)$ and $\sigma>0$, it yields $\left|I_{k}(x ; i t)\right| \leq \mu(\sigma+D+\varepsilon, F) e^{-\lambda_{k}(\sigma+D+\varepsilon)}$ and

$$
\begin{aligned}
& \int_{0}^{x} \exp \{(\sigma+i t) y\} \mathrm{d} \alpha(y)=\sum_{k=1}^{n-1}\left[\exp \left\{\lambda_{k+1} \sigma\right\} I_{k}\left(\lambda_{k+1} ; i t\right)\right. \\
& \left.-\sigma \int_{\lambda_{k}}^{\lambda_{k+1}} \exp \{\sigma y\} I_{k}(y ; i t) \mathrm{d} y\right] \\
& +\exp \{x \sigma\} I_{n}(x ; i t)-\sigma \int_{\lambda_{n}}^{x} \exp \{\sigma y\} I_{n}(y ; i t) \mathrm{d} y .
\end{aligned}
$$

In view of (5), there exists a constant $K$ such that $\lambda_{n}+$ $1 \leq \lambda_{n}+K$ for $n \geq 1$. So, we can deduce

$$
\begin{aligned}
& \left|\int_{0}^{x} \exp \{(\sigma+i t) y\} \mathrm{d} \alpha(y)\right| \\
& \leq \sum_{k=1}^{n-1} \mu(\sigma+D+\varepsilon, F) \exp \left\{-\lambda_{k}(\sigma+D+\varepsilon)\right\}\left(\exp \left\{\lambda_{k+1} \sigma\right\}+\left|\exp \left\{\lambda_{k+1} \sigma\right\}-\exp \left\{\lambda_{k} \sigma\right\}\right|\right) \\
& +\mu(\sigma+D+\varepsilon, F) \exp \left\{-\lambda_{n}(\sigma+D+\varepsilon)\right\}\left(\exp \{x \sigma\}+\left|\exp \{x \sigma\}-\exp \left\{\lambda_{n} \sigma\right\}\right|\right) \\
& \leq 2 \mu(\sigma+D+\varepsilon, F) e^{K \sigma} \sum_{k=1}^{n} \exp \left\{-\lambda_{k}(D+\varepsilon)\right\} \\
& =2 \mu(\sigma+D+\varepsilon, F) e^{K \sigma} \sum_{k=1}^{n} \exp \left\{-\lambda_{k}\left(D+\frac{1}{2} \varepsilon\right) \frac{D+\varepsilon}{D+(1 / 2) \varepsilon}\right\}
\end{aligned}
$$

In view of (5), for the above $\varepsilon$ and sufficiently large $n$, we have $\log n<\lambda_{n}(D+(1 / 2) \varepsilon)$,

$$
\exp \left\{-\lambda_{k}\left(D+\frac{1}{2} \varepsilon\right) \frac{D+\varepsilon}{D+(1 / 2) \varepsilon}\right\}<\frac{1}{n}^{(D+\varepsilon / D+(1 / 2) \varepsilon)},
$$

and $\sum_{k=1}^{+\infty}(1 / n)^{(D+\varepsilon / D+(1 / 2) \varepsilon)}$ is convergent. Therefore, this completes the proof of Lemma 4.

\section{Proofs of Theorems 2 and 3}

3.1. The Proof of Theorem 2. We firstly prove $\tau \leq T$. Assume that $T<+\infty$. From then for any positive number $\varepsilon$ and sufficiently large $\sigma$, it yields

$$
\log ^{+} M_{u}(\sigma, F)<e^{\sigma \rho(\sigma)}(T+\varepsilon)=\psi(r)(T+\varepsilon) .
$$

By Lemmas 2 and 4, it follows

$$
\begin{aligned}
& \log A_{n}^{*}<\psi(r)(T+\varepsilon)-\lambda_{n} \log r+\log 2 \\
& \leq \lambda_{n}\left\{\frac{1}{\rho(1+o(1))}-\log \varphi\left(\frac{\lambda_{n}}{(T+\varepsilon) \rho(1+o(1))}\right)\right\}, \\
& n \longrightarrow \infty .
\end{aligned}
$$

$$
T=\limsup _{\sigma \longrightarrow+\infty} \frac{\log ^{+} M_{u}(\sigma, F)}{e^{\sigma \rho(\sigma)}},
$$

Hence, in view of Lemma 1 , we can deduce 


$$
\begin{aligned}
\left(A_{n}^{*}\right)^{1 / \lambda_{n}} & \leq \frac{\exp (1 /(\rho(1+o(1))))}{\varphi\left(\lambda_{n} /((T+\varepsilon) \rho(1+o(1)))\right)} \\
& =\frac{\exp (1 /(\rho(1+o(1))))[\rho(T+\varepsilon)(1+o(1))]^{1 / \rho}}{\varphi\left(\lambda_{n}\right)}, \quad n \longrightarrow \infty,
\end{aligned}
$$

that is,

$$
\left[\varphi\left(\lambda_{n}\right)\left(A_{n}^{*}\right)^{1 / \lambda_{n}}\right]^{\rho} \leq \exp (1+o(1))[\rho(T+\varepsilon)(1+o(1))], \quad n \longrightarrow \infty .
$$

Since $\varepsilon$ is arbitrary, we conclude from the above inequality that

$$
\tau=\limsup _{n \longrightarrow+\infty} \frac{1}{\rho e}\left[\varphi\left(\lambda_{n}\right)\left(A_{n}^{*}\right)^{1 / \lambda_{n}}\right]^{\rho} \leq T,
$$

and $\tau<+\infty$ as $T<+\infty$.

Next, we prove $T \leq e^{D \rho} \tau$. Assume that $0 \leq \tau<+\infty$; then, for any $\varepsilon>0$, there exists a constant $K_{1}$ such that

$$
A_{n}^{*}<K_{1}\left\{\frac{[\rho e(\tau+\varepsilon)]]^{1 / \rho}}{\varphi\left(\lambda_{n}\right)}\right\}^{\lambda_{n}}, \quad n \geq 1
$$

Thus, from Lemma 3, Lemma 4, and (70), it yields

$$
\begin{aligned}
& \log M_{u}(\sigma, F) \leq \log C+\log K_{1}+K \sigma \\
&+\max _{n}\left\{\log \left[\frac{e^{\sigma+D+\varepsilon}[\rho e(\tau+\varepsilon)]^{1 / \rho}}{\varphi\left(\lambda_{n}\right)}\right]^{\lambda_{n}}\right\} \log M_{u}(\sigma, F) \\
& \leq \log C+\log K_{1}+K \sigma+\max _{n}\left\{\log \left[\frac{e^{\sigma+D+\varepsilon}[\rho e(\tau+\varepsilon)]^{1 / \rho}}{\varphi\left(\lambda_{n}\right)}\right]^{\lambda_{n}}\right\} \\
& \leq \log C+\log K_{1}+K \sigma+\frac{1}{\rho}(1+o(1)) \\
& \psi\left(e^{D+\varepsilon-\rho^{-1}(1+o(1))}[\rho e(\tau+\varepsilon)]^{1 / \rho} e^{\sigma}\right) \\
&= \log C+\log K_{1}+K \sigma+\frac{1}{\rho}(1+o(1)) e^{\rho(D+\varepsilon)+o(1)} \rho(\tau+\varepsilon) \psi\left(e^{\sigma}\right) \\
&= \log C+\log K_{1}+K \sigma \\
&+\left[e^{\rho(D+\varepsilon)+o(1)}(\tau+\varepsilon)+o(1)\right] \psi\left(e^{\sigma}\right), \quad(\sigma \longrightarrow+\infty),
\end{aligned}
$$

which implies

$$
\underset{\sigma \longrightarrow+\infty}{\limsup } \frac{\log M_{u}(\sigma, F)}{\psi\left(e^{\sigma}\right)} \leq e^{\rho(D+\varepsilon)}(\tau+\varepsilon) .
$$

Since $\varepsilon$ is arbitrary, let $\varepsilon \longrightarrow 0$ in (72); it follows that $T \leq e^{D \rho} \tau$ and $T<+\infty$ as $\tau<+\infty$.

Therefore, (16) is proved.

Furthermore, if the sequence $\left\{\lambda_{n}\right\}$ satisfies (18), then for any $\varepsilon \in\left(0, h^{\prime}\right)$, there exists a positive integer $n_{0}$ such that $\lambda_{n+1}-\lambda_{n}>h^{\prime}-\varepsilon$ for $n \geq n_{0}$. Thus, for any positive integer $k$, it follows

$$
\lambda_{k+n_{0}}-\lambda_{n_{0}}=\lambda_{k+n_{0}}-\lambda_{k-1+n_{0}}+\cdots+\lambda_{1+n_{0}}-\lambda_{n_{0}}>k\left(h^{\prime}-\varepsilon\right) .
$$

Hence,

$$
\frac{k+n_{0}}{\lambda_{k+n_{0}}}<\frac{\lambda_{k+n_{0}}-\lambda_{n_{0}}}{\lambda_{k+n_{0}}} \frac{1}{h^{\prime}-\varepsilon}+\frac{n_{0}}{\lambda_{k+n_{0}}} .
$$

Since $\varepsilon$ is arbitrary, then in view of (74), we conclude

$$
\begin{aligned}
D & =\underset{n \longrightarrow+\infty}{\limsup } \frac{\log n}{\lambda_{n}} \leq \limsup _{n \longrightarrow+\infty} \frac{\log n}{n} \limsup _{n \longrightarrow+\infty} \frac{n}{\lambda_{n}} \\
& \leq \limsup _{n \longrightarrow+\infty} \frac{\log n}{n} \limsup _{k \longrightarrow+\infty} \frac{k+n_{0}}{\lambda_{k+n_{0}}} \leq \frac{1}{h^{\prime}} \limsup _{n \longrightarrow+\infty} \frac{\log n}{n},
\end{aligned}
$$

that is, $D=0$. Thus, in view of (16), it follows $T=\tau=\lim \sup _{n \longrightarrow+\infty}(1 / \rho e)\left[\varphi\left(\lambda_{n}\right)\left(A_{n}^{*}\right)^{1 / \lambda_{n}}\right]^{\rho}$.

Therefore, this completes the proof of Theorem 2 .

3.2. The Proof of Theorem 3. We firstly prove $\tau_{p} \leq T_{p}$. Assume that $T_{p}<+\infty$. From

$$
T_{p}=\limsup _{\sigma \longrightarrow+\infty} \frac{\log _{p} M_{u}(\sigma, F)}{e^{\sigma \rho(\sigma)}},
$$

then for any positive number $\varepsilon$ and sufficiently large $\sigma$, it yields

$$
\log _{p} M_{u}(\sigma, F)<e^{\sigma \rho_{p}(\sigma)}\left(T_{p}+\varepsilon\right)=\psi_{p}(r)\left(T_{p}+\varepsilon\right),
$$

where $r=e^{\sigma}$. By Lemmas 2 and 4, for sufficiently large $\sigma$, it follows

$$
\begin{aligned}
\log A_{n}^{*} \leq & \log M_{u}(\sigma, F)-\lambda_{n} \sigma+\log 2<\exp _{p-1}\left\{\psi_{p}(r)\left(T_{p}+\varepsilon\right)\right\} \\
& -\lambda_{n} \log r+\log 2,
\end{aligned}
$$

where $\exp _{0}(x)=x, \exp _{p}(x)=\exp \left(\exp _{p-1}(x)\right),(p \geq 1)$. In view of Lemma 2, it is easy to see that $\exp _{p-1}\left\{\psi_{p}(r)\left(T_{p}+\varepsilon\right)\right\}-\lambda_{n} \log r+\log 2$ can attain the minimum when $r$ satisfies the following equation:

$$
r \psi_{p}^{\prime}(r) \prod_{j=1}^{p-1} \exp _{j}\left\{\left(T_{p}+\varepsilon\right) \psi_{p}(r)\right\}=\frac{\lambda_{n}}{T_{p}+\varepsilon} .
$$

By Lemma 1, we have $r \psi_{p}^{\prime}(r)=\psi_{p}(r) \rho_{p}(1+o(1))$ as $\sigma \longrightarrow+\infty$. Thus, it follows from (79) that

$$
\prod_{j=1}^{p-1} \exp _{j}\left\{\left(T_{p}+\varepsilon\right) \psi_{p}(r)\right\}=\frac{\lambda_{n}}{\rho_{p}(1+o(1))}, \quad n \longrightarrow+\infty \text {. }
$$

Hence, the function $\exp _{p-1}\left\{\psi_{p}(r)\left(T_{p}+\varepsilon\right)\right\}-\lambda_{n} \log r+$ $\log 2$ can obtain the minimum

$$
-(1+o(1)) \lambda_{n} \log \varphi_{p}\left(\frac{1}{T_{p}+\varepsilon} \log _{p-1} \frac{\lambda_{n}}{\rho_{p}(1+o(1))}\right), \quad n \longrightarrow+\infty,
$$

as $r$ satisfies equation (80). From $p \geq 2$ and Lemma 1 , it yields 


$$
\begin{gathered}
\varphi_{p}\left(\frac{1}{T_{p}+\varepsilon} \log _{p-1} \frac{\lambda_{n}}{\rho_{p}(1+o(1))}\right)=\varphi_{p}\left(\frac{1+o(1)}{T_{p}+\varepsilon} \log _{p-1} \lambda_{n}\right) \\
=\left(\frac{1+o(1)}{T_{p}+\varepsilon}\right)^{1 / \rho_{p}} \varphi_{p}\left(\log _{p-1} \lambda_{n}\right), \\
n \longrightarrow+\infty .
\end{gathered}
$$

So, we can deduce from (78), (81), and (82) that

$$
\begin{aligned}
\log A_{n}^{*} & \leq-(1+o(1)) \lambda_{n} \log \varphi_{p}\left(\frac{1}{T_{p}+\varepsilon} \log _{p-1} \frac{\lambda_{n}}{\rho_{p}(1+o(1))}\right) \\
& \leq-(1+o(1)) \lambda_{n} \log \left(\frac{1+o(1)}{T_{p}+\varepsilon}\right)^{1 / \rho_{p}} \varphi_{p}\left(\log _{p-1} \lambda_{n}\right), \\
n & \longrightarrow+\infty,
\end{aligned}
$$

that is,

$$
\left[\varphi_{p}\left(\log _{p-1} \lambda_{n}\right)\left(A_{n}^{*}\right)^{1 / \lambda_{n}}\right]^{\rho_{p}} \leq(1+o(1))\left(T_{p}+\varepsilon\right), \quad n \longrightarrow \infty .
$$

Since $\varepsilon$ is arbitrary, we conclude from the above inequality that

$$
\tau_{p}=\limsup _{n \longrightarrow+\infty}\left[\varphi_{p}\left(\log _{p-1} \lambda_{n}\right)\left(A_{n}^{*}\right)^{1 / \lambda_{n}}\right]^{\rho_{p}} \leq T_{p},
$$

and $\tau_{p}<+\infty$ as $T_{p}<+\infty$.

Next, we prove $T_{p} \leq e^{D \rho_{p}} \tau_{p}$. Assume that $0 \leq \tau_{p}<+\infty$; then, for any $\varepsilon>0$, there exists a constant $K_{2}$ such that

$$
A_{n}^{*}<K_{2}\left\{\frac{\left(\tau_{p}+\varepsilon\right)^{1 / \rho_{p}}}{\varphi_{p}\left(\log _{p-1} \lambda_{n}\right)}\right\}^{\lambda_{n}}, \quad n \geq 1 .
$$

Thus, from Lemma 3, Lemma 4, and (86), it yields

$$
\begin{aligned}
\log M_{u}(\sigma, F) \leq & \log C+\log K_{2}+K \sigma \\
& +\max _{n}\left\{\log \left[\frac{e^{\sigma+D+\varepsilon}\left[\left(\tau_{p}+\varepsilon\right)\right]^{1 / \rho_{p}}}{\varphi_{p}\left(\log _{p-1} \lambda_{n}\right)}\right]^{\lambda_{n}}\right\} .
\end{aligned}
$$

By using the same argument as in Lemma 3, we can conclude that the function

$$
\log \left[\frac{e^{\sigma+D+\varepsilon}\left[\left(\tau_{p}+\varepsilon\right)\right]^{1 / \rho_{p}}}{\varphi_{p}\left(\log _{p-1} \lambda_{n}\right)}\right]^{\lambda_{n}}
$$

can attain the maximum

$$
\frac{1+o(1)}{\rho_{p} \prod_{j=0}^{p-2} \log _{j} \lambda_{n} \exp _{p-1}\left(\psi_{p}\left((1+o(1)) \exp (\sigma+D+\varepsilon)\left(\tau_{p}+\varepsilon\right)^{1 / \rho_{p}}\right)\right), \quad n \longrightarrow+\infty}
$$

as $\lambda_{n}$ satisfies

$$
\log \left(\exp (\sigma+D+\varepsilon)\left(T_{p}+\varepsilon\right)^{1 / \rho_{p}}\right)-\log \varphi_{p}\left(\log _{p-1} \lambda_{n}\right)-\frac{1+o(1)}{\rho_{p} \prod_{j=0}^{p-2} \log _{j} \lambda_{n}=0, \quad n \longrightarrow+\infty}
$$
that

Thus, by Lemma 1, we can deduce from (87) and (89)

$$
\underset{\sigma \longrightarrow+\infty}{\limsup } \frac{\log _{p} M_{u}(\sigma, F)}{\psi_{p}\left(e^{\sigma}\right)} \leq e^{\rho_{p}(D+\varepsilon)}\left(\tau_{p}+\varepsilon\right) .
$$

Since $\varepsilon$ is arbitrary, let $\varepsilon \longrightarrow 0$ in (89); it follows that $T_{p} \leq e^{D \rho_{p}} \tau_{p}$ and $T_{p}<+\infty$ as $\tau_{p}<+\infty$. Hence, we prove (23).

Furthermore, if the sequence $\left\{\lambda_{n}\right\}$ satisfies (18), then we see $D=0$ from the proof of Theorem 2 . Thus, it is easy to prove that $T_{p}=\tau_{p}$ from (23).
Therefore, this completes the proof of Theorem 3.

\section{Proofs of Theorems 6 and 7}

4.1. The Proof of Theorem 6. Firstly of all, we prove $\tau_{a} \leq T$. Assume that $T<+\infty$. By using the same argument as in the proof of Theorem 2, for any positive number $\varepsilon$ and sufficiently large $\sigma$, we have (64). Since $F(s) \in L_{\infty}$, then we have $F(s) \in \bar{L}_{\beta}$ for any $\beta(-\infty<\beta<+\infty)$. Hence, from the definitions of $E_{n}(F, \beta)$ and $p_{n}$, it follows 


$$
\begin{aligned}
& E_{n}(F, \beta) \leq\left\|F-p_{n}\right\|_{\beta} \leq\left|F(\beta+i t)-p_{n}(\beta+i t)\right| \\
& \leq\left|\int_{0}^{+\infty} \exp \{(\beta+i t) y\} \mathrm{d} \alpha(y)-\int_{0}^{\lambda_{n}} \exp \{(\beta+i t) y\} \mathrm{d} \alpha(y)\right| \\
& =\left|\int_{\lambda_{n}}^{\infty} \exp \{(\beta+i t) y\} \mathrm{d} \alpha(y)\right|, \quad \beta<\sigma<+\infty .
\end{aligned}
$$

Let

$$
\begin{aligned}
& J(x ; \text { it })=\int_{0}^{x} \exp \{\text { it } y\} \mathrm{d} \alpha(y), \\
& J_{j+k}(b ; i t)=\int_{\lambda_{j+k}}^{b} \exp \{i t y\} \mathrm{d} \alpha(y), \\
& \left(\lambda_{j+k}<b \leq \lambda_{j+k+1}\right) .
\end{aligned}
$$

Then, $\left|J_{j+k}(b ; i t)\right| \leq A_{j+k}^{*}$. Since

$$
\begin{aligned}
& \left|\int_{\lambda_{k}}^{\infty} \exp \{(\beta+i t) y\} \mathrm{d} \alpha(y)\right| \\
& =\lim _{b \longrightarrow+\infty}\left|\int_{\lambda_{k}}^{b} \exp \{(\beta+i t) y\} \mathrm{d} \alpha(y)\right|, \quad-\infty<\beta<+\infty,
\end{aligned}
$$

then we can deduce

$$
\begin{aligned}
& \left|\int_{\lambda_{k}}^{b} \exp \{(\beta+i t) y\} \mathrm{d} \alpha(y)\right| \\
& =\left|\sum_{j=k}^{n+k-1} \int_{\lambda_{j}}^{\lambda_{j+1}} \exp \{\beta y\} d_{y} J_{j}(y ; i t)+\int_{\lambda_{n+k}}^{b} \exp \{\beta y\} d_{y} J_{n+k}(y ; i t)\right| \\
& =\mid\left[\sum_{j=k}^{n+k-1} e^{\lambda_{j+1} \beta} J_{j}\left(\lambda_{j+1} ; i t\right)-\beta \int_{\lambda_{j}}^{\lambda_{j+1}} e^{\beta y} J_{j}(y ; i t) \mathrm{d} y\right] \\
& \quad+e^{\beta b} J_{n+k}(b ; i t)-\beta \int_{\lambda_{n+k}}^{b} e^{\beta y} J_{j}(y ; i t) \mathrm{d} y \mid \\
& \quad \leq \sum_{j=k}^{n+k-1}\left[A_{j}^{*} e^{\lambda_{j+1} \beta}+A_{j}^{*}\left(e^{\lambda_{j+1} \beta}-e^{\lambda_{j} \beta}\right)\right] .
\end{aligned}
$$

Hence, from the above inequalities, we conclude

$$
\left|\int_{\lambda_{k}}^{\infty} \exp \{(\beta+i t) y\} \mathrm{d} \alpha(y)\right| \leq 2 \sum_{n=k}^{+\infty} A_{n}^{*} \exp \left\{\beta \lambda_{n+1}\right\}, \quad \text { as } n \longrightarrow+\infty \text {. }
$$

Thus, for any $\sigma(\beta<\sigma<+\infty)$, from (92) and (96) and by combining $A_{n}^{*} \leq 2 M_{u}(\sigma, F) e^{-\sigma \lambda_{n}}$, it yields

$$
E_{n}(F, \beta) \leq 2 \sum_{k=n+1}^{\infty} A_{k-1}^{*} \exp \left\{\beta \lambda_{k}\right\} \leq 4 M_{u}(\sigma, F) \sum_{k=n+1}^{\infty} \exp \left\{(\beta-\sigma) \lambda_{k}\right\} \text {. }
$$

Choosing $H(0<H<h)$ such that $\left(\lambda_{n+1}-\lambda_{n}\right) \geq H$ for $n \geq 0$, thus, for $\sigma \geq \beta+1$, we can deduce from (97) that

$$
\begin{aligned}
& E_{n}(F, \beta) \leq 4 M_{u}(\sigma, F) \exp \left\{\lambda_{n+1}(\beta-\sigma)\right\} \sum_{k=n+1}^{\infty} \exp \left\{\left(\lambda_{k}-\lambda_{n+1}\right)(\beta-\sigma)\right\} \\
& \leq 4 M_{u}(\sigma, F) \exp \left\{\lambda_{n+1}(\beta-\sigma)\right\} \exp \{H(n+1)\} \sum_{k=n+1}^{\infty}(\exp \{-H k\}) \\
& =4 M_{u}(\sigma, F) \exp \left\{\lambda_{n+1}(\beta-\sigma)\right\}(1-\exp \{H\})^{-1}
\end{aligned}
$$

that is,

$$
E_{n-1}(F, \beta) \leq K_{3} M_{u}(\sigma, F) \exp \left\{\lambda_{n}(\beta-\sigma)\right\}
$$

where $K_{3}$ is a constant.

Thus, it follows from (65) and (99) that

$\log \left[E_{n-1}(F, \beta) \exp \left(-\beta \lambda_{n}\right)\right]<\psi(r)(T+\varepsilon)-\lambda_{n} \log r+\log K_{3}$.

In view of (100) and using the same argument as in the proof of Theorem 2, we conclude from the above inequality that

$$
\tau_{a}=\limsup _{n \longrightarrow+\infty} \frac{1}{\rho \exp (\beta \rho+1)}\left[\varphi\left(\lambda_{n}\right)\left(E_{n-1}(F, \beta)\right)^{1 / \lambda_{n}}\right]^{\rho} \leq T,
$$

and $\tau_{a}<+\infty$ as $T<+\infty$.

Next, we prove $T \leq e^{D \rho} \tau_{a}$. Assume that $0 \leq \tau_{a}<+\infty$; then, for any $\varepsilon>0$, there exists a constant $K_{4}$ such that

$$
E_{n-1}(F, \beta) \exp \left(-\beta \lambda_{n}\right)<K_{4}\left\{\frac{\left[\rho e\left(\tau_{a}+\varepsilon\right)\right]^{1 / \rho}}{\varphi\left(\lambda_{n}\right)}\right\}^{\lambda_{n}}, \quad n \geq 1 .
$$

And since

$$
\begin{aligned}
A_{n}^{*} \exp \left\{\beta \lambda_{n}\right\}= & \sup _{\lambda_{n}<x \leq \lambda_{n+1},-\infty<t<+\infty}\left|\int_{\lambda_{n}}^{x} \exp \{i t y\} \mathrm{d} \alpha(y)\right| \exp \left\{\beta \lambda_{n}\right\} \\
& \leq \sup _{\lambda_{n}<x \leq \lambda_{n+1},-\infty<t<+\infty}\left|\int_{\lambda_{n}}^{x} \exp \{(\beta+i t) y\} \mathrm{d} \alpha(y)\right| \\
& \leq \sup _{-\infty<t<+\infty}\left|\int_{\lambda_{n}}^{\infty} \exp \{(\beta+i t) y\} \mathrm{d} \alpha(y)\right|
\end{aligned}
$$

thus, it means

$$
A_{n}^{*} \exp \left\{\beta \lambda_{n}\right\} \leq|F(\beta+i t)-p(\beta+i t)| \leq\|F-p\|_{\beta},
$$

for any $p \in \Pi_{n-1}$. In addition, there exists $p_{1} \in \Pi_{n-1}$ such that

$$
\left\|F-p_{1}\right\| \leq 2 E_{n-1}(F, \beta) .
$$

Thus, for any $\beta<+\infty$ and $F(s) \in L_{\infty}$, from (105) and (104), it yields

$$
A_{n}^{*} \exp \left\{\beta \lambda_{n}\right\} \leq 2 E_{n-1}(F, \beta) .
$$

Hence, in view of (102) and (106) and by using the same argument as in the proof of Theorem 2, we conclude

$$
\limsup _{\sigma \longrightarrow+\infty} \frac{\log M_{u}(\sigma, F)}{\psi\left(e^{\sigma}\right)} \leq e^{\rho(D+\varepsilon)}\left(\tau_{a}+\varepsilon\right),
$$

which implies $T \leq e^{D \rho} \tau_{a}$ and $T<+\infty$ as $\tau_{a}<+\infty$. Hence, (34) holds.

Furthermore, if the sequence $\lambda_{n}$ satisfies (18), from the proof of Theorem 2, it follows $D=0$, which means $T=\tau_{a}$.

Therefore, this completes the proof of Theorem 6 .

4.2. The Proof of Theorem 7. By using the same argument as in the proof of Theorem 3 and combining (99), (102), and (106), we can prove the conclusions of Theorem 7 easily. 


\section{Data Availability}

No data were used to support this study.

\section{Conflicts of Interest}

The authors declare that they have no conflicts of interest.

\section{Authors' Contributions}

H. Y. Xu was responsible for conceptualization and writing and preparing the original draft; H. Y. Xu and W. J. Tang contributed to writing the review and editing; and W. J. Tang, H. Y. Xu, and J. Chen were responsible for funding acquisition.

\section{Acknowledgments}

This work was supported by the National Natural Science Foundation of China (no. 11561033), the Natural Science Foundation of Jiangxi Province in China (nos. 20181BAB201001 and 20151BAB201008), and the Foundation of Education Department of Jiangxi (nos. GJJ190876, GJJ190895, and GJJ191042) of China.

\section{References}

[1] J. R. Yu, "Borel's line of entire functions represented by laplace-stieltjes transformation," Acta Mathematica Sinica, vol. 13A, pp. 471-484, 1963.

[2] D. V. Widder, The Laplace Transform, Princeton University Press, Princeton, NJ, USA, 1946.

[3] C. J. K. Batty, "Tauberian theorems for the Laplace-Stieltjes transform," Transactions of the American Mathematical Society, vol. 322, no. 2, pp. 783-804, 1990.

[4] X. Chang, S. Liu, P. Zhao, and X. Li, "Convergent predictioncorrection-based ADMM for multi-block separable convex programming," Journal of Computational and Applied Mathematics, vol. 335, pp. 270-288, 2018.

[5] X. Chang, S. Liu, and P. Zhao, "A note on the sufficient initial condition ensuring the convergence of directly extended 3block ADMM for special semidefinite programming," Optimization, vol. 67, no. 10, pp. 1729-1743, 2018.

[6] K. Knopp, "Über die konvergenzabszisse des Laplace-integrals," Mathematische Zeitschrift, vol. 54, no. 1951, pp. 291296, 1894.

[7] Y. Y. Kong and Y. Hong, On the Growth of Laplace-Stieltjes Transforms and the Singuar Direction of Complex Analysis, Jinan University Press, Guangzhou, China, 2010.

[8] Y. Y. Kong and Y. Y. Huo, "On generalized orders and types of Laplace-Stieltjes transformas analytic in the right half-plane," Acta Mathematica Sinica, vol. 59A, pp. 91-98, 2016.

[9] Y. Y. Kong and D. C. Sun, "On the growth of zero order Laplace-Stieltjes transform convergent in the right halfplane," Acta Mathematica Sinica, vol. 28B, no. 2, pp. 431-440, 2008.

[10] Y. Y. Kong and D. C. Sun, "On type-function and the growth of Laplace-Stieltjes transformations convergent in the right half-plane," Advances in Mathematics, vol. 37, no. 2, pp. 197-205, 2007, in Chinese.

[11] Y. Y. Kong, "The Laplace-Stieltjes transform of infinite order in the whole complex plane," Acta Mathematica Sinica, vol. 56A, no. 1, pp. 53-60, 2013.
[12] Y. Kong and Y. Yang, "On the growth properties of the Laplace-Stieltjes transform," Complex Variables and Elliptic Equations, vol. 59, no. 4, pp. 553-563, 2014.

[13] A. Mishkelyavichyus, "A Tauberian theorem for the LaplaceStieltjes integral and the dirichlet series (Russian)," Lithuanian Mathematical Journal, vol. 29, no. 4, pp. 745-753, 1989.

[14] M. Ru, "The recent progress in Nevanlinna theory," Journal of Jiangxi Normal University. Natural Sciences Edition, vol. 42, pp. 1-11, 2018.

[15] C. Singhal and G. S. Srivastava, "On the growth and approximation of entire functions represented by LaplaceStieltjes' transformation," Annali Dell'universita' Di Ferrara, vol. 63, no. 2, pp. 365-376, 2017.

[16] L. N. Shang and Z. S. Gao, "The growth of entire functions of infinite order represented by Laplace-Stieltjes transformation," Acta Mathematica Sinica, vol. 27A, no. 6, pp. 10351043, 2007.

[17] L. N. Shang and Z. S. Gao, "The value distribution of analytic functions defined by Laplace-Stieltjes transforms," Acta Mathematica Sinica, vol. 51A, no. 5, pp. 993-1000, 2008.

[18] H. Y. Xu, S. Y. Liu, and Q. P. Li, "Entire solutions for several systems of nonlinear difference and partial differential-difference equations of fermat-type," Journal of Mathematical Analysis and Applications, vol. 483, no. 2, Article ID 123641, 2020.

[19] H. Y. Xu, S. Y. Liu, and Q. P. Li, “The existence and growth of solutions for several systems of complex nonlinear difference equations," Mediterranean Journal of Mathematics, vol. 16, p. $8,2019$.

[20] H.-Y. Xu and Y.-Y. Kong, "The approximation of LaplaceStieltjes transformations with finite order on the left half plane," Comptes Rendus Mathematique, vol. 356, no. 1, pp. 63-76, 2018.

[21] H. Y. Xu and Y. Y. Kong, "The approximation of analytic function defined by Laplace- Stieltjes transformations convergent in the left half-plane," Houston Journal of Mathematics, vol. 43, pp. 783-806, 2017.

[22] H. Y. Xu and H. Wang, "The growth and approximation for an analytic function represented by Laplace-Stieltjes transforms with generalized order converging in the half plane," Journal of Inequalities and Applications, vol. 2018, Article ID 185, 2018.

[23] R. Ying and H. Y. Xu, "The growth of Hadamard product of random Dirichlet series," Jiangxi Normal University (Natural Science), vol. 43, pp. 513-517, 2019.

[24] H. S. Zhang and D. C. Sun, "The value distribution of LaplaceStieltjes transforms in the right half plane," Acta Mathematica Sinica, vol. 55A, no. 3, pp. 535-542, 2012.

[25] H. Y. Xu and S. Y. Liu, "The approximation of LaplaceStieltjes transforms with finite order," Journal of Inequalities and Applications, vol. 2017, no. 1, pp. 1-14, Article ID 164, 2017. 